\title{
Comparative Study between Platelet Rich Plasma and Adipose Derived Stem Cells in Periorbital Rejuvenation
}

\author{
N.W.Mikhael ${ }^{1}$, G.I.Elhabaa ${ }^{2}$, A.A.Shoulah ${ }^{2}$ and S.M.Megahed ${ }^{1}$ \\ ${ }^{1}$ Dermatology, Venereology and Andrology Dept., Faculty of Medicine, Benha Univ., Benha, Egypt \\ ${ }^{2}$ Plastic and Reconstructive Surgery, Dept., Faculty of Medicine, Benha Univ., Benha, Egypt \\ E-Mail:chaimaa.megahed@gmail.com
}

\begin{abstract}
Periorbital hyperpigmentation $(\mathrm{POH})$ is a commonly encountered worldwide problem that is characterized by relatively dark coloration around the eyelids. Its difficulty is due to its resistance to treatment, complex pathogenesis, and lacking straight forward long-term therapeutic options. both PRP and adipose-derived stem cells are therapeutic options that could be used as an alternative form of therapy alone or in combination with other conventional treatments of POH. Research has to focus on standardizing the PRP and ADSCs formulations and have a consensus data from the clinical trials from different research groups for better prognosis and to use as an alternative to conventional therapies. In this study, we compare the effect of platelet rich plasma injection, depending on the fact that platelets secrete growth factors and cytokines that have a positive influence on tissue healing and regeneration, versus the effect of adipose derived stem cells, that release important growth factors necessary for wound healing, immune system modulation and reducing inflammation. Regarding the results of this study, Both fat injection and PRP are effective treatments of POH and were associated with improvement. Better response, patients' satisfaction was significantly associated with fat injection when compared with PRP. Patients' satisfaction and physician's evaluation were significantly associated with improvement. Both procedures were tolerated and accepted by the patients. Both modalities are promising and may represent a good temporary solution for the problem of $\mathrm{POH}$.
\end{abstract}

Keywords: Platelet rich plasma, Adipose derived stem cells, Periorbital, Rejuvenation.

\section{Introduction}

Periorbital hyperpigmentation is a common dermatological problem and constitutes a significant percentage of patients visiting dermatology clinics. It has many synonyms as periocular hyperpigmentation, periorbital melanosis, dark circles, infraorbital darkening, and idiopathic cutaneous hyperchromia of the orbital region. Aging of the periocular area involves changes of the skin, muscles, fat, and bones. Facial fillers can be helpful in minimizing these changes by restoring youthful fullness to periocular areas that have undergone volume loss or loss of support[1].

Many facial plastic surgeons have sought to replace this volume with various injectable agents, both synthetic and autologous, in search for the ideal soft tissue filler. Injection of filler at infraorbital points could instantly lift the face up, elevating the point of shadow and shifting the point of highest light reflection to the ideal malar point [2].

Recent studies have shown the utility of adiposederived stem cells in the improvement of wound healing, describing their ability to regenerate soft tissues and their remodeling capacity provided by their unique cytokine and growth factor profiles. Despite ongoing concerns about survival and longevity of fat grafts after implantation and unpredictability of longterm outcome, fat has been successfully used as filler in many differ clinic situation. Adipose grafting has undergone significant changes over time. Many different techniques have been followed by trying to improve the quality of the lipoaspirate and the survival of the fat graft after implantation. A standard fat grafting technique is commonly performed in three stages: harvesting of adipose tissue from a suitable donor site; processing of the lipoaspirate to eliminate cellular debris, acellular oil and excess of infiltrated solution, reinjection of the purified adipose tissue. The most widely used surgical technique was described by Coleman. He modified and corrected the methods and results of his predecessors and proposed an atraumatic protocol for the treatment of adipose tissue. He reported that the key to successful fat grafting lies in the technique. In addition, he noticed that adipose tissue was not only a good filler, but improved the quality of the skin. In fact, fat grafts demonstrated to have not only dermal filler properties but also regenerative potential owing to the presence of stem cells in fat tissue [3].

Autologous fat transfer offers many qualities of ideal soft tissue filler. Main advantages of fat grafting ensue from the fact that the lipoaspirate tissue is an abundant source of regenerative pluripotent cells. However, the reported rates of fat cell survival vary greatly in the medical literature (10-90\%). Different techniques of harvesting, processing, and reinjecting the fat cells are so claimed to be responsible for these differences, without any agreement concerning the best way to process [4].

Platelet-rich plasma (PRP) is an emerging treatment in dermatology recently proposed for skin rejuvenation. PRP is an autologous concentration of human platelets contained in a small volume of plasma and has recently been shown to accelerate rejuvenate aging skin by various growth factors and cell adhesion molecules. It increases dermal collagen levels not only by growth 
factors, but also by skin needling (the mesotherapy technique 'point by point'). PRP application could be considered as an effective (even a single application) and safety procedure for facial skin rejuvenation. The clinical application of platelet-rich plasma (PRP) is based on the increase in the concentration of growth factors that are released from alpha-granule of the concentrated platelets and in the secretion of proteins which are able to capitalize on the healing process at the cellular level. It has been invented to restore the natural beauty by starting the natural rejuvenation process of the skin and aiming to make it function as a younger one and to keep the skin youthful and maintain it. PRP proved to promote wound healing and aid in facelift, volumetric skin, skin rejuvenation, regeneration, and reconstruction; improve wrinkling [5].

The ideal filler for aesthetic surgery is inexpensive and easy to obtain, natural in appearance and texture, immunologically compatible, and long lasting without risk of infection. By most metrics, autologous fat grafts meet these criteria perfectly. Although facial fat grafting is now a commonly accepted surgical procedure, there has been a wave of activity applying stem cells and platelet-rich plasma (PRP) therapies to aesthetic practice. It should neither be allergenic or carcinogenic nor susceptible to infections or biofilms, but still potentially reversible, temperature-stable, and cheap [6].

The escalating urge for a youthful-looking skin instigates continuous innovations with minimally invasive procedures. Advanced rejuvenation procedures for the upper face are becoming increasingly popular for aesthetic providers but are considered a high-risk treatment area for dermal filler/contouring products. Risks may range from bruising, which is manageable, to blindness, most often irreversible. Detailed comprehension of the facial anatomy is imperative when performing aesthetic injections including neuromodulators and dermal filler/contouring products. Understanding the location and function of the muscles, as well as landmarking the blood vessels and nerves, will assist the aesthetic provider to perform safe, confident injection procedures [7].

The aim of this work was to investigate and compare the efficacy of two modalities in the treatment of periorbital hyperpigmentation: platelet rich plasma (PRP) and adipose- derived stem cells (ADSCs).

\section{Patients and methods}

The study was conducted at Benha University Hospital, Dermatology and Venerology department, from January 2019 to March 2020. It included 30 female patients, aged from 18 years old to 52 years old, who attended the outpatient clinic.

This study included 30 patients, complaining of periorbital hyperpigmentation, fine wrinkles, eye bags and shadow effect in the periorbital area. They were be divided into 2 groups; (Group A) were injected with PRP and (Group B) were injected with ADSCs.

All patients were selected from the outpatient clinic of Dermatology and Venerology Department of Benha University Hospitals. Written informed consents were obtained from all participants. The study was be approved by the ethics committee on research involving human subjects of Benha Faculty of Medicine.

\section{Exclusion Criteria}

1- Patients suffering from ocular diseases.

2- Patients who had previous ocular or facial surgery.

3- Patients with autoimmune or hematologic disorders (bleeding or clotting disorders).

4- Patients who had previous cosmetic intervention in periorbital area.

5- Patients on anticoagulants such as aspirin, warfarin, etc.

6- Individuals with a history of syncope during or immediately after venipuncture either due to fear of injection or exaggerated vascular vasovagal tone.

7- Immunosuppression or patients under any kind of treatment causing absolute or relative immunosuppression.

8- Patients with any infectious disorder.

9- Patients with platelet count less than $100000 / \mathrm{ml}$.

10- Patients using systemic steroids.

11- Pregnant or lactating females.

12- Patients with chronic diseases such as chronic renal failure, hepatic insufficiency, cardiovascular disorders, diabetes mellitus, cancer, etc.

13- Patients involved in any other research project or clinical trial.

Diagnosis of $\mathrm{POH}$ was done in all patients based on detailed medical history (present history, past history and family history), clinical examination, laboratory tests (complete blood picture to exclude anemia) and dermoscopic evaluation.

PRP preparation: It was done according to the recommendation of Rachita Dhurat and MS Sukesh (2014) [8].

Adipose Derived-Stem Cells preparation:

- ADSCs preparation was done by microcannular tumescent liposuction technique using local anesthesia according to the recommendation of Jayashree V, Mysore V. (2007) [9].

- Sterilization of the donor area (lateral abdominal wall) was done by iodine the subcutaneous infiltration of local anesthesia was done using crystalloid fluid (xylocaine $2 \%$ with adrenaline diluted by lactated ringer's solution 1:10) (Klein's solution) for purposes of ballooning the fat tissue so as to decrease bleeding and for anesthesia.

- Then making small incisions about $2 \mathrm{~mm}$ to introduce microcannulae.

- Suction-assisted aspiration of fat by using small aspiration cannulae (microcannuale) $13-\mathrm{G}$ attached to $10 \mathrm{ml}$ syringe for fat harvesting. The unit was moved in and out while the plunger of the syringe 
was held in the retraction position. The syringe was then placed in an upside down position for 15 minutes and the non-fat portion was extruded.

\subsection{Statistical analysis}

The collected data were revised, coded, tabulated and introduced to a PC using Statistical package for Social Science (IBM Corp. Released 2017. IBM SPSS Statistics for Windows, Version 25. Armonk, NY: IBM Corp.). Data were presented and suitable analysis was done according to the type of data obtained for each parameter.

\section{Descriptive statistics}

Mean Standard deviation $( \pm \mathrm{SD}$ ) for parametric numerical data. Frequency and percentage of nonnumerical data.

\section{Analytical statistics}

Student $\mathrm{T}$ Test was used to assess the statistical significance of the difference between two study group means. Chi-Square test was used to examine the relationship between two qualitative variables. Fisher's exact test: was used to examine the relationship between two qualitative variables when the expected count is less than 5 in more than $20 \%$ of cells. Correlation analysis: Pearson's correlation was used to assess the strength of association between two quantitative variables. The correlation coefficient defines the strength and direction of the linear relationship between two variables. A $p$ value is significant if $\leq 0.05$ at confidence interval $95 \%$.

\section{Results}

The present study was conducted on 30 female cases complaining about periorbital hyperpigmentation, fine wrinkles, eye bags and shadow effect in the periorbital area. Their mean age was 29.8 years; $36.7 \%$ were healthcare workers, $23.3 \%$ were housewives, $20 \%$ were students, $13.3 \%$ were employee and $6.7 \%$ were teachers; $50 \%$ were single and $50 \%$ were married. None was smoker Table (1).

Table (1) Personal history of all studied cases.

\begin{tabular}{|c|c|c|c|c|}
\hline & & \multicolumn{3}{|c|}{ All cases } \\
\hline & & \multicolumn{3}{|c|}{$\mathrm{N}=\mathbf{3 0}$} \\
\hline \multirow[t]{3}{*}{ Age (years) } & & mean \pm SD & 29.8 & \pm 9.9 \\
\hline & Healthcare worker & $\mathbf{N}, \%$ & 11 & $36.7 \%$ \\
\hline & Housewife & $\mathrm{N}, \%$ & 7 & $23.3 \%$ \\
\hline \multirow[t]{3}{*}{ Occupation } & Student & $\mathbf{N}, \%$ & 6 & $20 \%$ \\
\hline & Employee & $\mathbf{N}, \%$ & 4 & $13.3 \%$ \\
\hline & Teacher & $\mathbf{N}, \%$ & 2 & $6.7 \%$ \\
\hline \multirow{2}{*}{ Marital status } & Single & $\mathbf{N}, \%$ & 15 & $50 \%$ \\
\hline & Married & $\mathbf{N}, \%$ & 15 & $50 \%$ \\
\hline Smoking & & $\mathbf{N}, \%$ & 0 & $0 \%$ \\
\hline
\end{tabular}

No significant differences were found regarding menopausal status, oral contraceptive pills intake or family history between studied groups. No significant differences were found regarding drug history and allergy between studied groups. Better patient's satisfaction, physician's evaluation and global aesthetic improvement scale were significantly associated with fat injection treatment when compared to PRP Table (2).

Table (2) Comparison of outcome between studied groups.

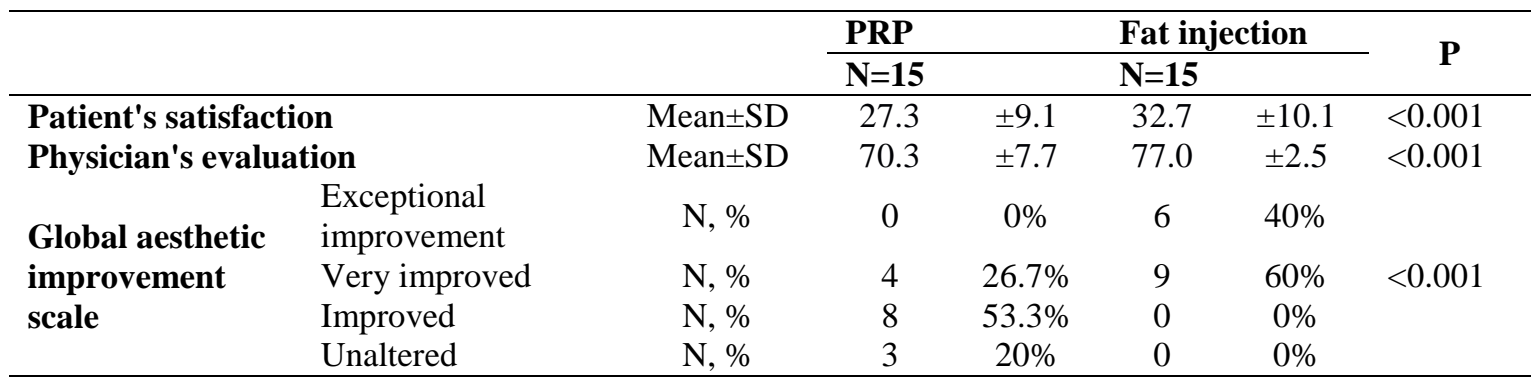

Better improvement was significantly associated with older age. Sleep, caffiene, sun exposure, stress, blue light exposure and hemoglobin concentration were not related to improvement degree by PRP Table (3). 
Table (3) Relation of improvement to baseline data in PRP group.

\begin{tabular}{|c|c|c|c|c|c|c|c|c|}
\hline \multirow[b]{3}{*}{ Age (years) } & \multirow[b]{3}{*}{ Mean \pm SD } & \multicolumn{6}{|c|}{ PRP } & \multirow{3}{*}{$\frac{\mathbf{P}}{0.039}$} \\
\hline & & \multicolumn{2}{|c|}{$\begin{array}{c}\text { Very improved } \\
N=4\end{array}$} & \multicolumn{2}{|c|}{$\begin{array}{c}\text { Improved } \\
\mathrm{N}=8\end{array}$} & \multicolumn{2}{|c|}{$\begin{array}{c}\text { Unaltered } \\
\quad \mathbf{N}=3 \\
\end{array}$} & \\
\hline & & 38.5 & \pm 12.6 & 23.6 & \pm 2.4 & 26 & \pm 6 & \\
\hline Irregular sleep habits & $\mathrm{N}, \%$ & 2 & $50 \%$ & 5 & $62.5 \%$ & 1 & $33.3 \%$ & 0.680 \\
\hline Caffeine intake & $\mathrm{N}, \%$ & 4 & $100 \%$ & 8 & $100 \%$ & 2 & $66.7 \%$ & 0.117 \\
\hline Sun exposure & $\mathrm{N}, \%$ & 0 & $0 \%$ & 2 & $25.0 \%$ & 0 & $0 \%$ & 0.364 \\
\hline Stress & $\mathrm{N}, \%$ & 2 & $50 \%$ & 6 & $75.0 \%$ & 1 & $33.3 \%$ & 0.405 \\
\hline Excess blue light exposure & $\mathrm{N}, \%$ & 0 & $0 \%$ & 2 & $25 \%$ & 0 & $0 \%$ & 0.364 \\
\hline Hemoglobin (g/dL) & Mean \pm SD & 11.2 & \pm 1.3 & 12.3 & \pm 1 & 13.1 & \pm 0.9 & 0.192 \\
\hline
\end{tabular}

No significant association was found between improvement and baseline data in fat injection group Table (4).

Table (4) Relation of improvement to baseline data in fat injection group.

\begin{tabular}{|c|c|c|c|c|c|c|}
\hline & & & Fat inject & & & \\
\hline & & Exc & $\begin{array}{l}\text { mprovement } \\
6\end{array}$ & Very & $\begin{array}{l}\text { iproved } \\
=9\end{array}$ & $\mathbf{P}$ \\
\hline Age (years) & Mean \pm SD & 27.2 & 8.6 & 34.3 & 10 & 0.174 \\
\hline Irregular sleep habits & $\mathrm{N}, \%$ & 2 & $33.3 \%$ & 4 & $44.4 \%$ & 0.667 \\
\hline Caffeine intake & $\mathrm{N}, \%$ & 6 & $100 \%$ & 7 & $77.8 \%$ & 0.486 \\
\hline Sun exposure & $\mathrm{N}, \%$ & 6 & $100 \%$ & 9 & $100 \%$ & - \\
\hline Stress & $\mathrm{N}, \%$ & 3 & $50 \%$ & 4 & $44.4 \%$ & 0.833 \\
\hline Excess blue light exposure & $\mathrm{N}, \%$ & 6 & $100 \%$ & 9 & $100.0 \%$ & - \\
\hline Hemoglobin (g/dL) & Mean \pm SD & 12.6 & 1 & 12.5 & 0.6 & 0.861 \\
\hline
\end{tabular}

Improvement showed significant positive correlation with patient's satisfaction and physician's evaluation in PRP and fat injection groups . In addition, improvement correlated significantly with age of onset in PRP in positive pattern and fat injection group in negative pattern . Otherwise, no significant correlations were found between improvement versus age or duration Table (5).

Table (5) Correlation of improvement with other studied parameters in studied groups.

\begin{tabular}{lcccc}
\hline & \multicolumn{4}{c}{ Improvement } \\
\cline { 2 - 5 } & \multicolumn{2}{c}{ PRP } & Fat injection \\
\cline { 2 - 5 } & $\mathbf{r}$ & $\mathbf{p}$ & $\mathbf{r}$ & $\mathbf{P}$ \\
\hline Age & 0.275 & 0.322 & -0.443 & 0.098 \\
Age of onset & 0.495 & 0.047 & -0.551 & 0.033 \\
Duration & -0.461 & 0.084 & -0.317 & 0.250 \\
Patient's satisfaction & 0.764 & 0.001 & 0.654 & 0.008 \\
Physician's evaluation & 0.925 & $<0.001$ & 0.958 & $<0.001$ \\
\hline
\end{tabular}

\section{Discussion}

The present study was conducted on 30 female cases complaining about periorbital hyperpigmentation, fine wrinkles, eye bags and shadow effect in the periorbital area. Their mean age was 29.8 years. No significant differences were found regarding age, occupation, address and marital status between studied groups. No significant differences were found regarding menopausal status, oral contraceptive pills intake or family history between studied groups. No significant differences were found regarding drug history and allergy between studied groups.

Better patient's satisfaction, physician's evaluation and Global aesthetic improvement scale were significantly associated with fat injection treatment when compared to PRP. Better improvement was significantly associated with older age. Sleep, caffeine, sun exposure, stress, blue light exposure and hemoglobin concentration were not related to improvement degree by PRP.

No significant association was found between improvement and baseline data in fat injection group. Better improvement was significantly associated with younger age of onset, radial pattern, pigmented vascular structural classes, while pigmented vascular class was significantly associated with very improved degree. Duration course, dermoscopic evaluation were not related to improvement degree by PRP. Patient's satisfaction and physician's evaluation were significantly associated with better improvement in fat injection group. 
Improvement showed significant positive correlation with patient's satisfaction and physician's evaluation in PRP and fat injection groups. In addition, improvement correlated significantly with age of onset in PRP in positive pattern and fat injection group in negative pattern. Otherwise, no significant correlations were found between improvement versus age, and duration.

Our results agreed with the study done by [10] from Iran. Their study investigated the effects of PRP as a possible rejuvenating agent for reduction in infraorbital dark circles. The study was conducted as a single session of $1.5 \mathrm{~mL}$ PRP injected intradermal into the tear trough area and wrinkles of crow's feet. They compared melanin content, stratum corneum hydration, and color homogeneity before and after injection. The improvement in infraorbital color homogeneity was statistically significant, but there was no statistically significant difference in melanin content and stratum corneum hydration. After 3 months of treatment, $80 \%$ of patients achieved fair to good improvement. They did not mention any information about excellent results. The authors stated that PRP may have the potential to improve infraorbital dark circle in terms of color homogeneity of the region, although this remains to be proven using larger, controlled studies.

Also our study can be compared to the study done by [11] where 3 sessions of autologous PRP were given into the periorbital area with 1 month apart between. The author reported fair improvement in $50 \%$, good response in $12 \%$, and excellent response in $6 \%$ and slight improvement in $32 \%$ of patients.

Also our study agreed with the study conducted by [12] that concluded that PRP is not the best method of treatment of $\mathrm{POH}$ as compared to chemical peeling. In their study they compared the effect of four sessions of chemical peeling using trichloroacetic acid and lactic acid with four sessions of PRP injection with 2 weeks of intervals in between sessions. Their study showed a significant improvement in favor of chemical peeling over PRP. Good improvement occurred in $47.6 \%$ in the group of chemical peeling versus $4.8 \%$ in the group who received PRP sessions $(\mathrm{P}<0.001)$. None of the PRP group had excellent improvement, while $38 \%$ of chemical peeling group did.

Our study agreed with the study done by [13] in which patients were subjected to seven intradermal injections of PRP on one periorbital side with 2 week intervals between sessions and carboxytherapy on the other side with 1 week interval apart. The study showed significant improvement in $\mathrm{POH}$ in both sides $(\mathrm{P} \leq .0001)$.The study concluded that both PRP and carboxytherapy relatively effective and their efficacy is comparable in treatment of $\mathrm{POH}$, but carboxtherapy is simple and slightly more effective modality and well tolerated than PRP.

Adipose tissue is now on the top one of stem cell sources regarding its accessibility, abundance, and less painful collection procedure when compared to other sources. Many reports showed that ADSCs-based cell therapy products demonstrated optimal efficacy and efficiency in some clinical indications for both autologous and allogeneic purposes, hence becoming considered as potential tools for replacing, repairing, and regenerating dead or damaged cells [14].

Different lipoaspirate centrifugation speeds and times are proposed. A study done by [15] illustrated that centrifugation speeds from $1000 \mathrm{rpm}$ to $4000 \mathrm{rpm}$ for 3 minutes showed no change in the percentage of adipocytes and adipose stem cell viability not only in the fresh samples but also in the cultured samples (1 day and 3 days). Centrifugation speeds under $4000 \mathrm{rpm}$ do not change the percentage of fat cell viability.

A study done by [16] demonstrated that centrifugation with a force greater than $50 \mathrm{~g}$ resulted in damage to the structural integrity of adipose tissue, increased necrosis and apoptosis of cells and decreased adipogenic differentiation capacity. Higher centrifugation speeds have also been correlated with increased fluid portion, reduced injectable tissue volume, and increased oil portion, which are associated with damage to adipocytes.

Another study done by [17] concluded that maximal long-term durability and fat cell viability results were obtained in the groups with $2000 \mathrm{rpm}$ or $447.2 \mathrm{G}$-force/4 minutes and $3000 \mathrm{rpm}$ or $1006.2 \mathrm{G}$ force/4 minutes centrifugation speed, indicating that 4 minutes centrifugation with an average G-force of 698.75 or $2500 \mathrm{rpm}$ provides the best results for the survival of autogenous fat grafts.

\section{Conclusion}

Both fat injection and PRP are effective treatments of $\mathrm{POH}$ and were associated with improvement. Better response, patients' satisfaction was significantly associated with fat injection when compared with PRP. Patients' satisfaction and physician's evaluation were significantly associated with improvement. Both procedures were tolerated and accepted by the patients. Both modalities are promising and may represent a good temporary solution for the problem of $\mathrm{POH}$.

\section{References}

[1] R. Sarkar and A. Das, "Periorbital hyperpigmentation: What lies beneath?," Indian Dermatol. Online J, Vol. 9(4), p. 229, 2018.

[2] R. Shetty, "Under eye infraorbital injection technique: the best value in facial rejuvenation," J. Cosmet. Dermatol, Vol. 13, no. 1, pp. 79-84, 2014.

[3] E. Bellini, M. P. Grieco, and E. Raposio, "The science behind autologous fat grafting," Ann. Med. Surg, Vol. 24, pp. 65-73, 2017.

[4] A. Modarressi, "Platlet rich plasma (PRP) improves fat grafting outcomes," World J. Plast. Surg, Vol. 2(1), p. 6, 2013.

[5] E. Elghblawi, "Platelet-rich plasma, the ultimate secret for youthful skin elixir and hair growth triggering," J. Cosmet. Dermatol, Vol. 17(3), pp. 423-430, 2018. 
[6] M. M. Aitzetmüller, I. Sukhova, G. M. Huemer, H.-G. Machens, J.-T. Schantz, and D. Duscher, "Soft tissue fillers: state of the art and future perspectives," Handchirurgie, Mikrochirurgie, Plast. Chir. Organ der Deutschsprachigen Arbeitsgemeinschaft fur Handchirurgie Organ der Deutschsprachigen Arbeitsgemeinschaft fur Mikrochirurgie der Peripher. Nerven und Gefasse Organ der V, Vol.49 (6), pp. 423-431, 2017.

[7] T. A. Hotta, "Understanding the anatomy of the upper face when providing aesthetic injection treatments," Plast. Surg. Nurs, Vol. 36(3), pp. 104-109, 2016.

[8] Dhurat R, Sukesh M. Principles and Methods of Preparation of Platelet-Rich Plasma: A Review and Author's Perspective. J Cutan Aesthet Surg. 2014.

[9] Jayashree V, Mysore V. Microcannular tumescent liposuction. Indian J Dermatol Venereol Leprol. 2007.

[10] P. Mehryan, H. Zartab, A. Rajabi, N. Pazhoohi, and A. Firooz, "Assessment of efficacy of platelet-rich plasma (PRP) on infraorbital dark circles and crow's feet wrinkles," J. Cosmet. Dermatol, Vol. 13(1), pp. 72-78, 2014.

[11] S. H. Al-Shami, "Treatment of periorbital hyperpigmentation using platelet-rich plasma injections," Am J Dermatol Venereol, Vol. 3(5), pp. 87-94, 2014.
[12] N. F. Ellabban, M. Eyada, H. Nada, and N. Kamel, "Efficacy and tolerability of using platelet-rich plasma versus chemical peeling in periorbital hyperpigmentation," J. Cosmet. Dermatol, Vol. 18( 6), pp. 1680-1685, 2019.

[13] Nofal E, Elkot R, Nofal A, Eldesoky F, Shehata S, Sami M. Evaluation of carboxytherapy and platelet-rich plasma in treatment of periorbital hyperpigmentation: A comparative clinical trial. J Cosmet Dermatol. 2018.

[14] L. Mazini, L. Rochette, M. Amine, and G. Malka, "Regenerative capacity of adipose derived stem cells (ADSCs), comparison with mesenchymal stem cells (MSCs),” Int. J. Mol. Sci, Vol. 20(10), p. 2523, 2019.

[15]D. Son, T. Choi, H. Yeo, J. Kim, and K. Han, "The effect of centrifugation condition on mature adipocytes and adipose stem cell viability," Ann. Plast. Surg, Vol. 72(5), pp. 589-593, 2014.

[16] Simonacci F, Bertozzi N, Grieco MP, Grignaffini E, Raposio E. Procedure, applications, and outcomes of autologous fat grafting. Ann Med Surg (Lond). 2017

[17] M. Bozkurt, E. Kapı, H. Şirinoğlu, E. Güvercin, G. T. Filinte, and D. Filinte, "The effects of the centrifugation speed on the survival of autogenous fat grafts in a rat model," J. Plast. Surg. Hand Surg, Vol. 50 (3), pp. 161-166, 2016. 